\title{
Síndrome de Burnout en la Facultad de Odontología de la Universidad Autónoma del Estado de México: un Estudio Comparativo
}

\author{
Burnout Syndrome in the Faculty of Dentistry, Universidad Autónoma del Estado de \\ Mexico: a Comparative Study
}

Angel Visoso Salgado*; Paola Ariselda Sánchez Reyna** \& Norma Margarita Montiel Bastida**

VISOSO, S. A.; SÁNCHEZ, R. P. A.; MONTIEL, B. N. M. Síndrome de Burnout en la facultad de odontología de la universidad autónoma del estado de méxico: un estudio comparativo. Int. J. Odontostomat., 6(2):129-138, 2012.

RESUMEN: EI Síndrome de Burnout (SBO) es un problema creciente, comprende alteraciones físicas, somáticas y psicológicas; así como ausentismo laboral, y cuantiosas pérdidas económicas. Desafortunadamente presenta alta prevalencia en los profesionales de la salud y en los estudiantes, principalmente los del área odontológica. El objetivo de este trabajo fue conocer las diferencias por afectación del SBO, en tres grupos (docentes, trabajadores administrativos y estudiantes) que conforman el personal de la Facultad de Odontología, de la Universidad Autónoma del Estado de México (UAEM). Se aplicó el Maslach Burnout Inventory y un cuestionario sobre las características sociodemográficas, laborales y académicas, a 108 personas de la Facultad de Odontología, de la UAEM: 36 docentes, 36 trabajadores administrativos y 36 estudiantes. El instrumento consta de 22 ítems para evaluar las tres subescalas del SBO: 1) Agotamiento Emocional (AE), 2)Despersonalización (DP), y 3) Realización Personal (RP). Se aplicó una prueba t para conocer las diferencias entre los grupos, con apoyo del software SPSS, versión 19. Al comparar las medias de afectación por el SBO entre los grupos, se encontraron diferencias estadísticamente significativas en la subescala $A E$ entre docentes y estudiantes ( $p=0,00)$, entre docentes y trabajadores administrativos $(p=0,01)$, y entre trabajadores administrativos y estudiantes $(p=0,00)$. En la subescala DP sólo se encontraron diferencias entre los trabajadores administrativos y los estudiantes $(p=0,01)$. En la subescala RP se encontraron diferencias entre los docentes y los estudiantes $(p=0,00)$, entre los docentes y los trabajadores administrativos $(p=0,01)$, y entre los trabajadores administrativos y los estudiantes $(p=0,05)$. No hay estudios sobre el SBO, que incluyan todos los grupos que conforman el personal de una Facultad de Odontología. Sin embargo, es importante conocer las diferencias en la afectación por este síndrome en cada uno de los grupos, lo que permitirá la elaboración de estrategias específicas para cada uno, y evitar en lo posible sus consecuencias físicas, psicológicas y sociales.

PALABRAS CLAVE: Síndrome de Burnout, odontólogo, enfermedad profesional.

\section{INTRODUCCIÓN}

El término "Burn out" se originó en el lenguaje de los deportistas anglosajones, cuya traducción al castellano significa "estar quemado". Fuera de este ámbito, se utilizó este término en la década de los 70 para referirse a los problemas de fatiga y cansancio profesional de los servicios socio-sanitarios. Sin embargo, se ha constatado que todos los profesionales, sea cual sea su ocupación, son susceptibles de desanimarse y perder su entusiasmo por su trabajo (Mondol López, 2008).
En 1974, Herbert F. (Freudenberger, 1974) en un hospital de Nueva York, observó que muchos de sus compañeros, al cabo del año de trabajo sufrían una gran desmotivación y una progresiva pérdida de la energía hasta llegar al agotamiento. Lo denominó el Síndrome del quemado según la expresión original del inglés (Burnout síndrome): SBO, terminología que dos años después acuñó la psicóloga social Cristine Maslach, quien en conjunto con Susana Jackson, en 1981 lo definieron como "un síndrome de cansancio

* Dr. en C.S.P. Profesor Investigador del Centro de Investigación y Estudios Avanzados en Odontología, Facultad de Odontología de la Universidad Autónoma del Estado de México, México.

" M. en C.O. Profesor de Asignatura de la Facultad de Odontología de la Universidad Autónoma del Estado de México, México.

*** Dra. en O. Coordinadora del Centro de Investigación y Estudios Avanzados en Odontología, Facultad de Odontología de la Universidad Autónoma del Estado de México, México. 
emocional, despersonalización y baja realización personal, que puede ocurrir entre individuos que trabajan con personas". Además de describir las tres dimensiones o componentes del síndrome: 1) Cansancio emocional: en el sentido de desgaste, pérdida de energía, agotamiento y fatiga; 2) despersonalización o deshumanización: que consiste en el desarrollo de aptitudes negativas hacia otras personas, sobre todo con las que se trabaja, junto a la irritabilidad y a la pérdida de motivación; y 3) falta de realización personal, es decir, actitud negativa hacia sí mismo y hacia su trabajo, con baja moral, baja productividad laboral, poca autoestima e incapacidad para soportar el estrés (Maslach \& Jackson, 1997; Cáceres Bermejo, 2006).

Así, el SBO se ha transformado en un problema creciente, que abarca alteraciones físicas, somáticas y psicológicas, pudiendo llevar a depresión, ausentismo laboral recurrente, y causante de millonarias pérdidas (Cartagena Pizarro \& Nunez Franz, 2003); así como exceso en el consumo de cafeína, tabaco, estimulantes o tranquilizantes, ingesta hipercalórica, hipersódica o hiperlípida), generando cansancio emocional como la expresión básica del SBO que se relaciona directamente con el estrés académico (Extremera et al., 2007).

La presentación del Síndrome de Burnout, desafortunadamente tiene una gran prevalencia en los profesionales de la salud (Gil-Monte \& Peiró, 1997); incluyendo a los estudiantes de dicha área (Sanders, 2002); y dentro de esta área, los odontólogos son los profesionales de la salud mayormente afectados por el Síndrome de Burnout, donde factores como: el clima organizacional, factores organizacionales, aunados al tipo de demandas de las propias actividades, los roles interpersonales, y la ambigüedad de los mismos entre otros, son factores que generan y/o desencadenan el desgaste emocional (Díaz Romero et al., 2006). Sumado a que se ha documentado que la práctica dental es considerada como la profesión de las ciencias de la salud que más ansiedad produce (Algar et al., 1998).

\section{MATERIAL Y MÉTODO}

Se aplicó el Maslach Burnout Inventory (MBI) (Maslach \& Jackson, 1981, 1986; Cáceres Bermejo), así como un cuestionario encaminado a conocer las características sociodemográficas, laborales y acadé- micas, a 108 personas de la Facultad de Odontología de la Universidad Autónoma del Estado de México: 36 docentes, 36 trabajadores administrativos y 36 estudiantes de licenciatura y posgrado.

Se utilizó el MBI, en su versión validada en castellano (Moreno et al., 1991), diseñado para valorar el SBO en sus tres dimensiones o subescalas del Síndrome de Burnout: 1) Cansancio Emocional (CE), 2) Despersonalización (DP), y 3) Realización Personal (RP). Está compuesto por 22 ítems. Para las respuestas se utilizó una escala de Likert con puntuaciones de 0 a 6 . La subescala de cansancio emocional está compuesta por 9 ítems $(1,2,3,6,8,13,14,16,20)$ y mide los sentimientos de sobrecarga emocional. La subescala de despersonalización estuvo integrada por 5 ítems $(5,10,11,15,22)$ y valoró los sentimientos y actitudes negativas hacia el paciente. La subescala de realización personal constó de 8 ítems $(4,7,9,12$, $17,18,19,21)$, y midió sentimientos de competencia y de consecución de logros (Tabla I).

La aplicación del MBI y del cuestionario sobre las características sociodemográficas, laborales y académicas de los individuos, se realizó previo consentimiento informado, de forma personalizada a cada uno de los que participaron en el presente estudio, en un cubículo aislado, con luz natural, y sin distractores tanto en el interior como en el exterior.

Las mediciones de la afectación del SBO en los diferentes grupos de estudio se realizaron en dos etapas:

Mediciones de los niveles de afectación en cada grupo de estudio considerando la escala propuesta por Moreno y Caballero (Moreno et al., 2002; Caballero et al., 2001):

Cansancio emocional: bajo (-18) - Medio (19-26) Alto (27 o más)

Despersonalización: Bajo (-5) - Medio (6-9) - Alto (10 o más)

Realización personal: Bajo (40 o más) - Medio (34-39) - Alto (33 o menos)

Para la comparación entre los tres grupos de estudio, se consideraron los puntajes obtenidos en el $\mathrm{MBI}$, para así poder realizar el análisis estadístico correspondiente encaminado a conocer la diferencia entre las medias de afectación por el SBO. Lo anterior con la aplicación de una prueba t, con apoyo del software SPSS, en su versión 19. 


\section{MASLACH BURNOUTINVENTORY}

\begin{tabular}{|c|c|c|c|c|c|c|}
\hline \multicolumn{7}{|c|}{ Frecuencia } \\
\hline 0 & 1 & 2 & 3 & 4 & 5 & 6 \\
\hline Nunca & $\begin{array}{c}\text { Pocas veces } \\
\text { al año o } \\
\text { menos }\end{array}$ & $\begin{array}{c}\text { Una vez al } \\
\text { mes o menos }\end{array}$ & $\begin{array}{l}\text { Unas pocas } \\
\text { veces al mes }\end{array}$ & $\begin{array}{c}\text { Una vez a la } \\
\text { semana }\end{array}$ & $\begin{array}{c}\text { Pocas veces a } \\
\text { la semana }\end{array}$ & Todos los días \\
\hline
\end{tabular}

Marcar con una cruz la casilla correspondiente según la numeración de la tabla anterior

Me siento emocionalmente agotado por mi trabajo

Me siento cansado al final de lajornada de trabajo

Me siento fatigado cuando me levanto por la mañana y tengo que enfrentarme con otro día de trabajo

Fácilmente comprendo cómo se sienten los pacientes

Creo que trato a algunos pacientes como si fueran objetos impersonales

Trabajar todo el día con mucha gente es un esfuerzo

Trato muy eficazmente los problemas de los pacientes

Me siento quemado por mi trabajo

Creo que estoy influyendo positivamente con mi trabajo en las vidas de otras personas

Me he vuelto más insensible con la gente desde que ejerzo esta profesión

Me preocupa el hecho de que este trabajo me esté endureciendo mucho emocionalmente

Me siento muy activo

Me siento frustrado en mi trabajo

Creo que estoy trabajando demasiado

Realmente no me preocupa lo que ocurre a algunos de mis pacientes

Trabajar directamente con las personas me produce estrés

Me siento estimulado después de trabajar en contado con mis pacientes

Fácilmente puedo crear una atmósfera relajada con mis pacientes

He conseguido muchas cosas útiles en mi profesión

Me siento acabado

En mi trabajo, trato los problemas emocionales con mucha calma

Siento que los pacientes me culpan por algunos de sus problemas

\begin{tabular}{|c|c|c|c|c|c|c|}
\hline 0 & 1 & 2 & 3 & 4 & 5 & 6 \\
\hline 0 & 1 & 2 & 3 & 4 & 5 & 6 \\
\hline 0 & 1 & 2 & 3 & 4 & 5 & 6 \\
\hline 0 & 1 & 2 & 3 & 4 & 5 & 6 \\
\hline 0 & 1 & 2 & 3 & 4 & 5 & 6 \\
\hline 0 & 1 & 2 & 3 & 4 & 5 & 6 \\
\hline 0 & 1 & 2 & 3 & 4 & 5 & 6 \\
\hline 0 & 1 & 2 & 3 & 4 & 5 & 6 \\
\hline 0 & 1 & 2 & 3 & 4 & 5 & 6 \\
\hline 0 & 1 & 2 & 3 & 4 & 5 & 6 \\
\hline 0 & 1 & 2 & 3 & 4 & 5 & 6 \\
\hline 0 & 1 & 2 & 3 & 4 & 5 & 6 \\
\hline 0 & 1 & 2 & 3 & 4 & 5 & 6 \\
\hline 0 & 1 & 2 & 3 & 4 & 5 & 6 \\
\hline 0 & 1 & 2 & 3 & 4 & 5 & 6 \\
\hline 0 & 1 & 2 & 3 & 4 & 5 & 6 \\
\hline 0 & 1 & 2 & 3 & 4 & 5 & 6 \\
\hline 0 & 1 & 2 & 3 & 4 & 5 & 66 \\
\hline 0 & 1 & 2 & 3 & 4 & 5 & 6 \\
\hline 0 & 1 & 2 & 3 & 4 & 5 & 6 \\
\hline 0 & 1 & 2 & 3 & 4 & 5 & 6 \\
\hline 0 & 1 & 2 & 3 & 4 & 5 & 6 \\
\hline
\end{tabular}

\section{RESULTADOS Y DISCUSIÓN}

La edad promedio de los grupos considerados en el presente estudio (docentes: $57,28 \pm 8,02$ ) (estudiantes: $22,72 \pm 5,25$ )(trabajadores administrativos:
$22,72 \pm 5,25$ ) (Tabla I), difiere a la edad reportada en estudios previos sobre el SBO 46,58 $\pm 9,47$ años (Varela-Centelles et al., 2005), y 43,30 $\pm 9,79$ años 
VISOSO, S. A.; SÁNCHEZ, R. P. A.; MONTIEL, B. N. M. Síndrome de Burnout en la facultad de odontología de la universidad autónoma del estado de méxico: un estudio comparativo. Int. J. Odontostomat., 6(2):129-138, 2012.

Tabla II. Características sociodemográficas, laborales y académicas de los individuos estudiados.

\begin{tabular}{|c|c|c|c|c|c|c|c|c|c|c|c|c|c|c|c|}
\hline \multicolumn{16}{|c|}{ Variables continuas } \\
\hline \multirow[b]{2}{*}{ Variables } & \multicolumn{5}{|c|}{ Docentes } & \multicolumn{5}{|c|}{ Estudiantes } & \multicolumn{5}{|c|}{ Administrativos } \\
\hline & $\mathrm{n}$ & Media & $\mathrm{DE}$ & Min & Máx & $\mathrm{n}$ & Media & $\mathrm{DE}$ & Mín & Máx & $\mathrm{n}$ & Media & $\mathrm{DE}$ & Mín & Máx \\
\hline Edad & 36 & 57.28 & 8.02 & 40 & 67 & 36 & 22.72 & 5.25 & 20 & 40 & 36 & 39.50 & 15.65 & 22 & 62 \\
\hline No de hijos & 36 & 2.00 & .48 & 1 & 3 & 36 & .17 & .51 & 0 & 2 & 36 & 1.25 & .84 & 0 & 2 \\
\hline Antigüedad laboral & 36 & 29.39 & 5.06 & 20 & 39 & 36 & 1.58 & .65 & 1 & 3 & 36 & 3.69 & 4.02 & 1 & 18 \\
\hline $\begin{array}{l}\text { Tiempo de exposición } \\
\text { a estresores }\end{array}$ & 13 & 6.92 & 1.04 & 6 & 8 & 27 & 4.33 & 1.39 & 2 & 6 & 9 & 8.00 & .00 & 8 & 8 \\
\hline $\begin{array}{l}\text { Tiempo de actividades } \\
\text { académicas }\end{array}$ & 34 & 4.88 & .77 & 4 & 6 & 2 & 3.00 & .00 & 3 & 3 & 0 & 0 & .00 & 0 & 0 \\
\hline
\end{tabular}

Variables categóricas

\begin{tabular}{|c|c|c|c|c|c|c|c|}
\hline \multirow{2}{*}{ Variable } & \multirow{2}{*}{ Categorías } & \multicolumn{2}{|c|}{ Docentes } & \multicolumn{2}{|c|}{ Estudiantes } & \multicolumn{2}{|c|}{ Administrativos } \\
\hline & & Frecuencia & $\%$ & Frecuencia & $\%$ & Frecuencia & $\%$ \\
\hline \multirow{2}{*}{ Sexo } & Femenino & 8 & 22.22 & 28 & 77.78 & 27 & 75.00 \\
\hline & Masculino & 28 & 77.78 & 8 & 22.22 & 9 & 25.00 \\
\hline \multirow{6}{*}{ Escolaridad } & Secundaria & & & & & 16 & 44.80 \\
\hline & Preparatoria & & & & & 17 & 49.60 \\
\hline & Licenciatura & 16 & 44.44 & 36 & 100.00 & 2 & 5.60 \\
\hline & Especialidad & 10 & 27.78 & & & & \\
\hline & Maestría & 6 & 16.67 & & & & \\
\hline & Doctorado & 4 & 11.11 & & & & \\
\hline \multirow{3}{*}{ Estado Civil } & Casado & 30 & 83.34 & 4 & 11.11 & 18 & 50.00 \\
\hline & Soltero & 4 & 11.11 & 32 & 88.88 & 18 & 50.00 \\
\hline & Viuda & 2 & 5.56 & & & & \\
\hline \multirow{2}{*}{ Relaciones Laborales } & Buenas & 12 & 33.33 & 20 & 55.56 & & \\
\hline & Regulares & 24 & 66.67 & 16 & 44.44 & 36 & 100.00 \\
\hline \multirow{8}{*}{ Estresores } & Injusticias & 2 & 5.56 & & & & \\
\hline & Ruido & 8 & 22.22 & 3 & 8.33 & & \\
\hline & Pacientes & & & 28 & 77.78 & & \\
\hline & Jefe & & & 2 & 5.56 & 9 & 25.00 \\
\hline & Calor & & & 1 & 2.78 & & \\
\hline & Emergencias & & & 2 & 5.56 & & \\
\hline & Todos & 4 & 11.11 & & & & \\
\hline & Ninguno & 22 & 61.11 & & & 27 & 75.00 \\
\hline \multirow{2}{*}{ Actividades Académicas } & Si & 34 & 94.44 & 36 & 100.00 & 0 & 0.00 \\
\hline & No & 2 & 5.56 & 0 & 0.00 & 36 & 100.00 \\
\hline \multirow{3}{*}{ Tipo de Actividades Académicas } & Clínica & 16 & 44.44 & 34 & 94.44 & & \\
\hline & Docencia & 18 & 50.00 & 2 & 5.56 & & \\
\hline & Ninguno & 2 & 5.56 & & & 36 & 100.00 \\
\hline \multirow{2}{*}{ Otro Trabajo } & $\mathrm{Si}$ & 28 & 77.78 & 2 & 5.56 & & \\
\hline & No & 8 & 22.22 & 34 & 94.44 & 36 & 100.00 \\
\hline \multirow{4}{*}{ Tipo de Trabajo } & Director & 4 & 11.11 & & & & \\
\hline & Practica Privada & 24 & 66.67 & & & & \\
\hline & Docencia & & & 2 & 5.56 & & \\
\hline & Ninguno & 8 & 22.22 & 34 & 94.44 & 36 & 100.00 \\
\hline
\end{tabular}


(Cáceres Bermejo). Lo anterior puede ser debido a que los estudios realizados previamente se han realizado en odontólogos de la práctica privada a diferencia de los de este trabajo donde se estudiaron a odontólogos dedicados a la docencia, y los estudios realizados en estudiantes de odontología reportan una edad de 20 años (Hernández et al., 2007; Albanesi de Nasetta et al., 2006; Aranda et al., 2003) similar a la del presente estudio. No existe edad reportada para trabajadores administrativos en una facultad de odontología.

En relación al sexo (Tabla II), la mayoría de los estudios realizados muestran que el SBO se presenta mayormente en mujeres (Albadalejo et al., 2004; Atance, 1997; Robles Velásquez \& Cáceres Gutiérrez, 2010; Cedeño \& Brandt, 2002; Gil-Monte, 2002), lo que concuerda con los resultados del presente estudio sólo para el grupo de los alumnos y los trabajadores administrativos, ya que en el grupo de los docentes predominó el sexo masculino concordando con lo reportado en otros estudios pero en odontólogos de la práctica clínica y no en odontólogos docentes como en el presente estudio (Caballero et al.; Prieto et al., 2002; Quiroz \& Saco, 1999; Varela-Centelles et al.; Cáceres Bermejo). Respecto al sexo en el grupo de los estudiantes de odontología, el sexo masculino es predominante en lo reportado (Seisdedos, 1997), información contraria a la del presente estudio, donde para este grupo el sexo predominante fue el femenino. Aunque en investigación realizada en México en estudiantes, se encontraron porcentajes similares entre hombres y mujeres (Preciado-Serrano \& VázquezGoñi, 2010).

El número de hijos promedio (Tabla II), para los docentes fue de 2, en los estudiantes fue de 0,17 y en los administrativos de 1,25. Información que concuerda con lo reportado (2 hijos) (Cáceres Bermejo), pero sólo para el caso de los docentes. Otros estudios han estudiado la asociación entre el tener hijos y la presencia del SBO (Sánchez-Madrid et al., 2005; Simón García et al., 2005).

La antigüedad laboral (Tabla II), en el grupo de los docentes fue de $29,39 \pm 5,06$, para el grupo de los estudiantes de 1,58 $\pm 0,65$ y para el grupo de los trabajadores administrativos fue de $3,69 \pm 4,02$ años. Datos discordantes con lo reportado en la literatura de estudio realizados en odontólogos, donde la media de antigüedad laboral fue de 15,25 \pm 8,34 (Varela-Centelles et al.), y 19 años (Cáceres Bermejo), información que podría deberse a que en el presente estudio se estu- dió a odontólogos docentes, y lo reportado en la literatura es en odontólogos clínicos; donde las expectativas sin duda cambian en el tiempo en dichos grupos, ya que algunos autores han encontrado que el SBO sería más frecuente después de los diez primeros años de ejercicio profesional, de forma que tras este período ya se habría producido la transición de las expectativas idealistas a la práctica cotidiana, viéndose como las recompensas profesionales y económicas no son, ni las prometidas, ni las esperadas (Hillhouse \& Adler, 1997). Sin embargo, otros estudios han encontrado que los primeros años de trabajo serían los más vulnerables para el desarrollo del síndrome, de forma que a medida que aumenta la experiencia profesional disminuiría el cansancio emocional (Albar et al., 2004).

El tiempo de exposición a estresores (Tabla II), reportado por el grupo de los docentes fue de $6,92 \pm 1,04$, los estudiantes reportaron $4,33 \pm 1,39$, y los trabajadores administrativos reportaron $8 \pm 0$ horas de exposición. Sin embargo, los estudios reportados sólo han estudiado la asociación del estrés en odontólogos (Lartigue \& Fernández, 1998) o estudiantes (Rajab, 2001) con el SBO, sin cuantificar el tiempo de exposición al estrés en los individuos estudiados.

Respecto al tiempo de actividades académicas (Tabla II), reportado por los docentes, trabajadores administrativos y estudiantes del presente estudio, no existe información en la literatura consultada.

La escolaridad reportada en los tres grupos de estudio (Tabla II), no es posible compararla con estudios previos, debido a que sólo existen datos de la asociación de la escolaridad y la presencia del SBO en odontólogos (Díaz Romero et al., 2006; Osborne \& Croicher, 1994).

Los porcentajes obtenidos sobre el estado civil (Tabla II), de los integrantes de los grupos estudiados, no es posible compararlos del todo, ya que sólo en el caso de los estudiantes existe en la literatura un estudio sobre este rubro, donde el $95 \%$ eran solteros y sólo el $5 \%$ casados, datos similares a lo encontrado en el presente estudio donde en el grupo de los estudiantes el $88,88 \%$ reportó ser soltero y el $11,11 \%$ casados. Otros estudios sólo han reportado una asociación positiva entre el estado civil de los odontólogos y la presencia del SBO (Díaz Romero et al.).

Respecto al tipo de actividades académicas (Tabla II), el tipo de relaciones al interior de la facultad, y el tipo de estresores reportados por los integrantes de 
los tres grupos estudiados, no existen estudios que reporten dichos rubros en personas con SBO.

Los resultados obtenidos sobre los niveles de afectación en los tres grupos de estudio por el SBO en cada una de las subescalas que lo componen, mostraron que en el grupo de los docentes en la escala de AE presentaban un nivel de afectación bajo, en la escala de DP un nivel medio y en la escala de RP un nivel bajo (Tabla III). Sin embargo, los estudios realizados al respecto, no han abordado este grupo de estudio en el área odontológica; sólo han estudiado la presencia de SBO en odontólogos de la práctica clínica (Robles Velásquez \& Cáceres Gutiérrez; Albanesi de Nasetta et al.; Leggat et al., 2001; Díaz Romero et al.; Osborne \& Croicher). Por lo que la información obtenida no puede ser contrastada.

En el grupo de los estudiantes, se encontró en la escala de AE un nivel alto, en la escala DP un nivel medio y en la escala RP un nivel medio (Tabla III). Información concordante con lo reportado: donde el Agotamiento Personal fue la subescala predominantemente afectada, seguida de la subescala de DP y de RP (Albanesi de Nasetta et al.; Rajab) por otro lado, los datos difieren de lo reportado en otro estudio, donde la subescala predominantes fue la de RP, seguida de la DP y AE (Preciado-Serrano \& Vázquez-Goñi).

El grupo de trabajadores administrativos presentó un nivel de afectación bajo en la escala de $\mathrm{AE}$, un nivel bajo en DP y un nivel alto en RP (Tabla III). Sobre este grupo estudiado, no existen reportes de afectación del SBO en las escalas que lo comprenden.

Considerando los tres grupos comprendidos en el estudio (docentes, trabajadores administrativos y estudiantes) se encontró un nivel de afectación bajo en la escala de $\mathrm{AE}$, un nivel de afectación medio en la escala de DP y un nivel de afectación medio en la escala de RP (Tabla III). Sin embargo, no existe información reportada al respecto en los tres grupos estudiados para tener un punto de referencia.

Al comparar las medias entre grupos por la afectación del SBO, en la escala de $\mathrm{AE}$, se encontraron diferencias estadísticamente significativas entre los docentes y los estudiantes $(p=0,00)$ con predominio para estos últimos (Tabla IV). Información concordante con lo reportado en estudio realizado sobre el SBO entre estudiantes y odontólogos (Albanesi de Nasetta et al.).

De igual forma, se encontraron diferencias sig- nificativas entre los docentes y los trabajadores administrativos $(p=0,01)$, y entre los estudiantes y trabajadores administrativos $(p=0,00)$ (Tabla IV). No existiendo información reportada al respecto para contrastar los datos obtenidos en el presente estudio.

En la subescala DP se encontraron diferencias estadísticamente significativas sólo entre los estudiantes y los trabajadores administrativos $(p=0,01)$ (Tabla IV). Sin embargo no existen estudios que comparen la afectación por el SBO entre estos grupos.

Respecto a la subescala de RP se encontraron diferencias estadísticamente significativas entre los docentes y estudiantes, con mayor afectación para los estudiantes $(p=0,00)$ (Tabla IV). Información acorde a lo reportado entre estos grupos de estudio (Albanesi de Nasetta et al.).

Respecto a la comparación en la afectación por el SBO en la subescala de RP entre docentes y trabajadores administrativos y entre los estudiantes y los trabajadores administrativos. No existen datos que nos permitan realizar una posible comparación de los mismos. Sin embargo, existe estudio realizado sólo en el grupo de los estudiantes, donde la mayor afectación la presentaron en RP, seguida de DP y AE (PreciadoSerrano \& Vázquez-Goñi).

Al comparar cada uno de los grupos estudiados contra el grupo total (los tres grupos de estudio), en la escala de agotamiento emocional se encontró diferencias estadísticamente significativas entre el grupo de los docentes y el grupo total $(p=0,05)$, estudiantes y grupo total $(p=0,00)$, y grupo total contra trabajadores administrativos $(p=0,00)$. En la escala de despersonalización se encontraron significancias entre el grupo de los estudiantes y el grupo total $(p=0,01)$ y al comparar el grupo de los administrativos y el grupo total $(p=0,03)$; en esta escala no se encontraron diferencias entre el grupo de los docentes y el grupo total. En la escala de realización personal se encontraron diferencias entre el grupo de los docentes y el grupo total $(p=0,00)$, y entre el grupo de los trabajadores administrativos y el grupo total $(p=0,02)$; no encontrándose diferencias entre el grupo de los estudiantes y el grupo total (Tabla V).

Respecto a las diferencias encontradas al comparar las medias de afectación por el SBO en cada uno de los grupos estudiados contra el grupo total (los tres grupos de estudio), no existe información reportada sobre el tema para saber si existe concordancia o no con los resultados obtenidos. 
VISOSO, S. A.; SÁNCHEZ, R. P. A.; MONTIEL, B. N. M. Síndrome de Burnout en la facultad de odontología de la universidad autónoma del estado de méxico: un estudio comparativo. Int. J. Odontostomat., 6(2):129-138, 2012.

Tabla III Síndrome de Burnout en el personal de la Facultad de Odontología de la Universidad Autónoma del Estado de México.

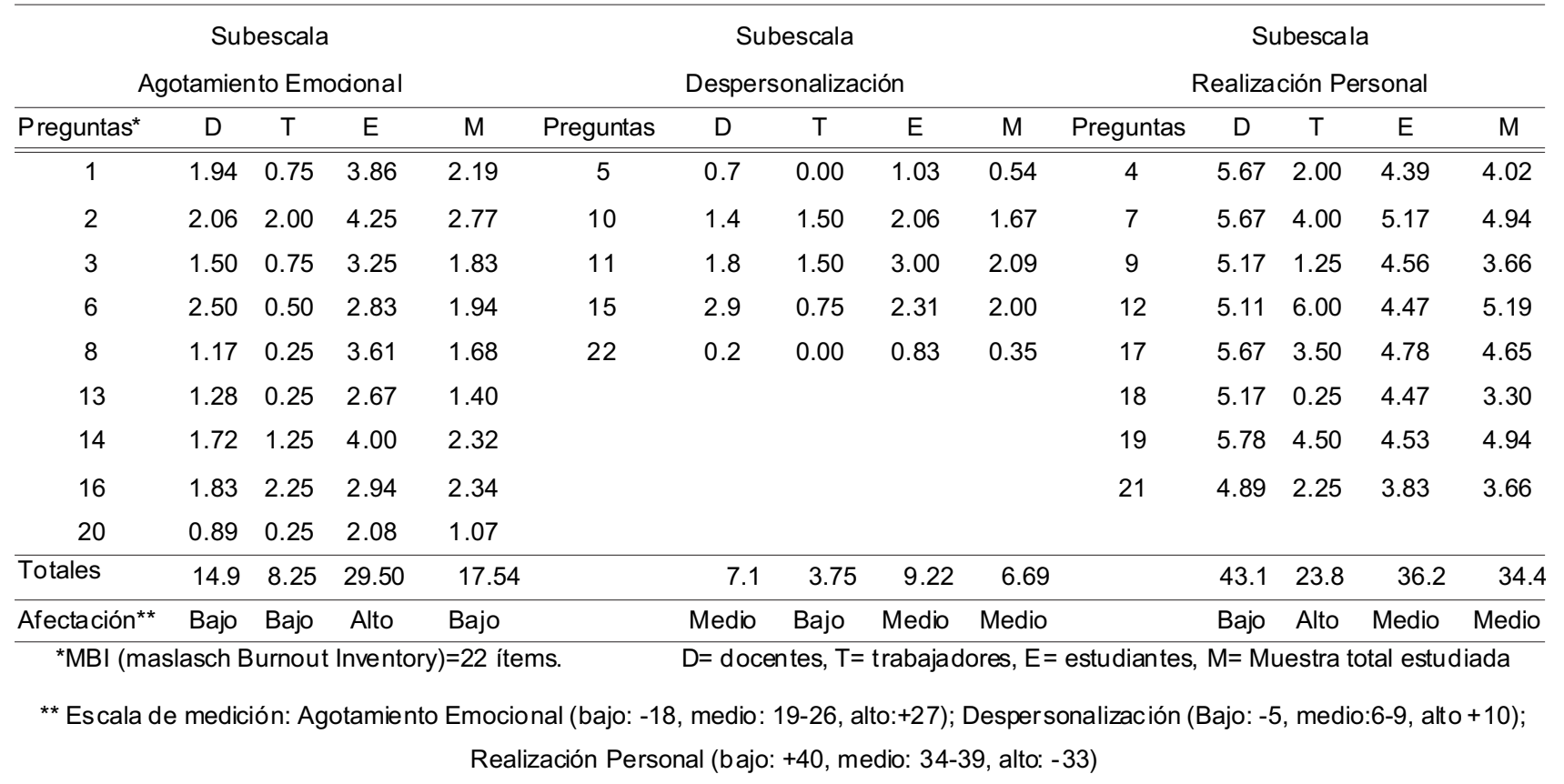

Tabla IV. Diferencia de medias entre grupos, de acuerdo al nivel de afectación del Síndrome de Burnout.

\begin{tabular}{|c|c|c|c|c|c|c|}
\hline Subescalas & Grupos de Estudio & Media & DE & Diferencia de Medias & $t$ & $\mathrm{p}$ \\
\hline \multirow{6}{*}{ Agotamiento Emocional } & Docentes & 1.65 & 0.50 & \multirow[t]{2}{*}{-1.62} & \multirow[t]{2}{*}{-7.15} & \multirow[t]{2}{*}{$0.00^{* *}$} \\
\hline & Estudiantes & 3.28 & 0.71 & & & \\
\hline & Docentes & 1.65 & 0.50 & \multirow[t]{2}{*}{0.74} & \multirow[t]{2}{*}{3.21} & \multirow[t]{2}{*}{$0.01^{* *}$} \\
\hline & Administrativos & 0.92 & 0.76 & & & \\
\hline & Estudiantes & 3.28 & 0.71 & \multirow[t]{2}{*}{2.36} & \multirow[t]{2}{*}{9.12} & \multirow{2}{*}{$0.00^{* *}$} \\
\hline & Administrativos & 0.92 & 0.76 & & & \\
\hline \multirow{6}{*}{ Despersonalización } & Docentes & 1.33 & 0.89 & \multirow[t]{2}{*}{-0.52} & \multirow[t]{2}{*}{-2.26} & \multirow[t]{2}{*}{0.09} \\
\hline & Estudiantes & 1.85 & 0.91 & & & \\
\hline & Docentes & 1.33 & 0.89 & \multirow[t]{2}{*}{0.58} & \multirow[t]{2}{*}{1.84} & \multirow[t]{2}{*}{0.14} \\
\hline & Administrativos & 0.75 & 0.75 & & & \\
\hline & Estudiantes & 1.85 & 0.91 & \multirow[t]{2}{*}{1.10} & \multirow[t]{2}{*}{5.69} & \multirow[t]{2}{*}{$0.01^{* *}$} \\
\hline & Administrativos & 0.75 & 0.75 & & & \\
\hline \multirow{6}{*}{ Realización personal } & Docentes & 5.39 & 0.34 & \multirow[t]{2}{*}{0.87} & \multirow[t]{2}{*}{8.13} & \multirow[t]{2}{*}{$0.00^{* *}$} \\
\hline & Estudiantes & 4.53 & 0.38 & & & \\
\hline & Docentes & 5.39 & 0.34 & \multirow[t]{2}{*}{2.42} & \multirow[t]{2}{*}{3.78} & \multirow[t]{2}{*}{0.01 ** } \\
\hline & Administrativos & 2.97 & 1.88 & & & \\
\hline & Estudiantes & 4.53 & 0.38 & \multirow[t]{2}{*}{1.56} & \multirow[t]{2}{*}{2.43} & \multirow[t]{2}{*}{$0.05^{*}$} \\
\hline & Administrativos & 2.97 & 1.88 & & & \\
\hline
\end{tabular}

$\mathrm{DE}=$ Desviación Estándar. $\quad{ }^{*} \mathrm{p} \leq 0.05,{ }^{* *} \mathrm{p} \leq 0.01$ 
Tabla V. Diferencia de medias entre cada grupo vs el grupo total de estudio, de los niveles de afectación del Síndrome de Burnout.

\begin{tabular}{|c|c|c|c|c|c|c|}
\hline Subescalas & Grupos de Estudio & Media & $\mathrm{DE}$ & Diferencia de Medias & $\mathrm{t}$ & $\mathrm{p}$ \\
\hline \multirow{6}{*}{$\begin{array}{l}\text { Agotamiento } \\
\text { Emocional }\end{array}$} & Docentes & 1.65 & 0.50 & \multirow[t]{2}{*}{-0.29} & \multirow[t]{2}{*}{-2.34} & \multirow[t]{2}{*}{$0.04^{*}$} \\
\hline & Grupo Total & 1.95 & 0.52 & & & \\
\hline & Estudiantes & 3.28 & 0.71 & \multirow[t]{2}{*}{1.33} & \multirow[t]{2}{*}{9.32} & \multirow[t]{2}{*}{$0.00^{* *}$} \\
\hline & Grupo Total & 1.95 & 0.52 & & & \\
\hline & Administrativos & 0.92 & 0.76 & \multirow[t]{2}{*}{-1.03} & \multirow[t]{2}{*}{-7.13} & \multirow[t]{2}{*}{$0.00^{* *}$} \\
\hline & Grupo Total & 1.95 & 0.52 & & & \\
\hline \multirow{6}{*}{ Despersonalización } & Docentes & 1.33 & 0.89 & \multirow[t]{2}{*}{-0.01} & \multirow[t]{2}{*}{-0.06} & \multirow[t]{2}{*}{0.96} \\
\hline & Grupo Total & 1.34 & 0.82 & & & \\
\hline & Estudiantes & 1.85 & 0.91 & \multirow[t]{2}{*}{0.51} & \multirow[t]{2}{*}{4.86} & \multirow[t]{2}{*}{$0.01^{* *}$} \\
\hline & Grupo Total & 1.34 & 0.82 & & & \\
\hline & Administrativos & 0.75 & 0.75 & \multirow[t]{2}{*}{-0.59} & \multirow[t]{2}{*}{-3.21} & \multirow[t]{2}{*}{$0.03^{*}$} \\
\hline & Grupo Total & 1.34 & 0.82 & & & \\
\hline \multirow{6}{*}{$\begin{array}{l}\text { Realización } \\
\text { personal }\end{array}$} & Docentes & 5.39 & 0.34 & \multirow[t]{2}{*}{1.10} & \multirow[t]{2}{*}{5.01} & \multirow[t]{2}{*}{$0.00^{* *}$} \\
\hline & Grupo Total & 4.30 & 0.72 & & & \\
\hline & Estudiantes & 4.53 & 0.38 & \multirow[t]{2}{*}{0.23} & \multirow[t]{2}{*}{1.05} & \multirow[t]{2}{*}{0.33} \\
\hline & Grupo Total & 4.30 & 0.72 & & & \\
\hline & Administrativos & 2.97 & 1.88 & \multirow[t]{2}{*}{-1.33} & \multirow[t]{2}{*}{-3.11} & \multirow[t]{2}{*}{$0.02^{*}$} \\
\hline & Grupo Total & 4.30 & 0.72 & & & \\
\hline
\end{tabular}

$\mathrm{DE}=$ Desviación Estándar. $\quad$ * $\mathrm{p} \leq 0.05,{ }^{* *} \mathrm{p} \leq 0.01$

Los estudios previos sólo han abordado el SBO categorizándolo de acuerdo a los niveles de afectación del mismo; sin embargo, no se han realizado comparaciones entre grupos contemplando cada una de las subescalas que lo comprenden: motivo del presente estudio. Además de que no se han abordado los tres grupos (docentes, estudiantes y trabajadores administrativos) de una facultad de odontología, lo que hace compleja la discusión de los resultados obtenidos. Lo anterior, es de llamar la atención, ya que es sabido que dentro del área de la salud, el área odontológica es la que mayormente se presenta el SBO.

La escasez de información al respecto, hace necesaria la investigación que incorpore a todos los grupos de una facultad de odontología, ya que el conocer la afectación por el SBO en cada uno de ellos, permitirá realizar intervenciones para cada grupo en específico, encaminadas a la mejor funcionalidad del entorno general de una facultad de odontología.

Sobre todo, si consideramos las consecuencias adversas del SBO, que por un lado provocan trastornos ansiosos o depresivos, consumo de sustancias, aparición de adicciones, y trastornos cardiovasculares, y por el otro aumentan el ausentismo laboral, los de- seos de abandonar el trabajo, disminución de la productividad y de la eficacia, así como disminución de la satisfacción laboral.

Repercusiones que se deben tomar en cuenta si consideramos que los profesionales que se dedican a cuidar a otras personas están afectados por este síndrome, y por lo tanto las consecuencias no sólo las tendrán de manera particular, sino afectaran a la sociedad en su conjunto.

VISOSO, S. A.; SÁNCHEZ, R. P. A.; MONTIEL, B. N. M. Burnout syndrome in the faculty of dentistry, universidad autónoma del estado de mexico: a comparative study. Int. J. Odontostomat., 6(2):129-138, 2012.

ABSTRACT: Burnout syndrome (BOS) is a growing problem, involves physical, somatic and psychological alterations, as well as work absenteeism, and economic losses. Unfortunately it has high prevalence among health professionals and students, mainly on dentistry area. The aim of this study was to know the differences by affectation of the BOS, between the three groups (educational, administrative workers and students) that conforms the personnel of the Dentistry Faculty, of the Autonomous University of the State of Mexico (UAEM). The Maslach Burnout Inventory and a questionnaire on sociodemographic, 
labor and academic characteristics, was applied to 108 people of the Dentistry Faculty of the UAEM: 36 educational ones, 36 administrative workers and 36 students. The instrument includes 22 items to evaluate the three subscales of the BOS: 1) Emotional fatigue (EF), 2) Depersonalization (DP), and 3) Personal Realization (PR). A t test was applied to know the differences between the groups studied, with support of software SPSS, version 19. When comparing the averages of affectation by BOS between the groups studied, were statistically significant differences in the subscale of EF between the educational and students groups $(p=0.00)$, between educational and administrative workers groups $(p=0.01)$, and between administrative workers and students groups $(p=0.00)$. In the subscale of DP only were differences between the administrative workers and students groups $(p=0.01)$. In the subscale of PR were differences between educational and students groups $(p=0.00)$, between educational and administrative workers $(p=0.01)$ and, between administrative workers and students groups $(p=0.05)$. There are not studies on the BOS that includes all the groups that conform the personnel of a Dentistry Faculty. Nevertheless, it is important to know the differences in the affectation by this syndrome in each one of the groups, since it will allow elaborating specific strategies for each one; and on this way avoid the physical, psychological and social consequences that cause.

KEY WORDS: Burnout syndrome, dentist, occupational disease.

\section{REFERENCIAS BIBLIOGRÁFICAS}

Albadalejo, R.; Villanueva, R.; Ortega, P.; Astasio, P.; Calle, M. E. \& Domínguez, V. Síndrome de Burnout en personal de enfermería de un hospital de Madrid. Rev. Esp. Salud Pública, 78(4):505-16, 2004.

Albanesi de Nasetta, S.; Tifner, S. \& Nasetta, J. Estrés en odontología. Acta Odontol. Venez., 44(3):310-5, 2006.

Albar, M. J.; Romero, M.; González, M. D.; Carballo, E.; García, A.; Gutiérrez, I. \& Algaba, S. Apoyo social, características sociodemográficas y burnout en enfermeras y auxiliares de hospital. Enferm. Clín., 14(5):281-5, 2004.

Algar, P. J.; González Sanz, A.; Gil González, F. J.; Miguel Tobal, F. \& Martín Díaz, M. D. Ansiedad y estrés en la profesión dental. Prof. Dental, 1(3):39-42, 1998.

Aranda, C.; Pando, M.; Brizuela, I.; Acosta, M. \& Reyes, M. Síndrome de burnout y factores psicosociales en el trabajo en estudiantes de postgrado del Departamento de Salud Pública de la Universidad de
Guadalajara, México. Rev. Psiquiatria Fac. Med. Barna, 30(4):193-9, 2003.

Atance, J. C. Aspectos epidemiológicos del síndrome de burnout en personal sanitario. Rev. Esp. Salud Pública, 71(3):294-303, 1997.

Caballero, M. A.; Bermejo, F.; Nieto, R. \& Caballero, F. Prevalencia y factores asociados al burnout en un área de salud. Aten. Prim., 27(5):313-7, 2001.

Cartagena Pizarro, H. A. \& Nunez Franz, M. L. Síndrome de Burnout en odontólogos del nivel primario de atencion adscritos a la direccion comunal de salud de Talca. Memoria de pregrado Odontología, Universidad de Talca, Talca, Chile, 2003.

Cáceres Bermejo, G. G. Prevalencia del Síndrome de Burnout en personal sanitario militar. Tesis Doctoral, Universidad Complutense de Madrid, Madrid, España, 2006.

Cedeño, E. \& Brandt, C. Síndrome de Burnout en los residentes de postgrado de medicina familiar y en los médicos familiares en Venezuela. Med. Fam. (Caracas), 10(2):91-99, 2002.

Díaz Romero, R. M.; Pérez Romero, E. \& Lartigue Becerra, M. T. Desgaste profesional en un grupo de odontólogos. Rev. ADM, 63(6):220-4, 2006.

Extremera, N.; Durán, A. \& Rey, L. Inteligencia emocional y su relación con los niveles de burnout, engagement y estrés en estudiantes universitarios. Rev. Educ., 342:239-56, 2007.

Freudenberger, H. Staff Burn-Out. J. Social Issues, 30(1):159-65, 1974.

Gil-Monte, P. Influencia del género sobre el proceso de desarrollo del síndrome de quemarse por el trabajo (Burnout) en profesionales de enfermería. Psicol. Estud., 7(1):3-10, 2002.

Gil-Monte, P. \& Peiró, J. Desgaste psíquico en el trabajo: el síndrome de quemarse. Madrid, Síntesis, 1997. pp.3-14.

Hernández, C. P.; Monroy, A.; Rivera, P.; Rojas, B. E. \& Sánchez, N. S. Estudio comparativo de los síntomas del síndrome de burnout entre odontólogos de práctica general y especialistas del Distrito Federal y área Metropolitana en el 2007. Disponible en: http:/ /odontologia.iztacala.unam.mx/instrum_y_lab1/otros/ COLO-QUIOXIX 
VISOSo, S. A.; SÁNCHEZ, R. P. A.; MONTIEL, B. N. M. Síndrome de Burnout en la facultad de odontología de la universidad autónoma del estado de méxico: un estudio comparativo. Int. J. Odontostomat., 6(2):129-138, 2012.

Hillhouse, J. J. \& Adler, C. M. Investigating stress effect patterns in hospital staff nurses: results of a cluster analysis. Soc. Sci. Med., 45(12):1781-8, 1997.

Lartigue, T. \& Fernández, V. Enfermería, una profesión de alto riesgo. México, Plaza Valdés Editores, 1998.

Leggat, P. A.; Chowanadisai, S.; Kedjarune, U.; Kukiattrakoon, B. \& Yapong, B. Health of dentists in southern Thailand. Int. Dent. J., 51(5):348-52, 2001.

Maslach, C. \& Jackson, S. The measurement of experienced burnout. J. Ocup. Behav., 2(2):99-113, 1981.

Maslach, C.; Jackson, S. E. Maslach Burnout Inventory Manual. 2nd ed. Palo Alto, CA, Consulting Psychologist Press, 1986.

Maslach, C. \& Jackson, S. Maslach Burnout Inventory. En: Seis dedos, N. (Ed.). Manual del Inventario Burnout de Maslach. Síndrome del "quemado" por estrés laboral asistencial. Madrid, TEA Ediciones, S.A., 1997.

Mondol López, M. Análisis de la prevalencia del Síndrome de Burnout en los odontólogos y sus repercusiones en la salud del profesional y en la atención bucodental del Servicio de Odontología en la Clínica Carlos Durán durante el período Agosto - Noviembre del 2007. Tesis de Maestria, Instituto Centroamericano de Administración Pública, San José, Costa Rica, 2008.

Moreno, B.; Gónzalez, J. L.; Garrosa, E. \& Peñacoba, C. Desgaste profesional en hospitales. Influencia de las variables sociodemográficas. Rev. Rol Enferm., 25(11):18-26, 2002.

Moreno, B.; Oliver, C.; Pastor, J. C. \& Aragoneses, A. El Burnout una forma específica de estrés laboral. En: Carballo, V. E. \& Buela, G. (Eds.). Manual de Psicología Clínica. Madrid, Siglo XXI, 1991. pp.271-84.

Osborne, D. \& Croicher, R. Leis of Burnout in general dental practitioners in the South East or England. Br. Dent., 177:372-7, 1994.

Preciado-Serrano, M. L. \& Vázquez-Goñi, J. M. Perfil de estrés y síndrome de Burnout en estudiantes mexicanos de odontología de una universidad pública. Rev. Chil. Neuro-Psiquiat., 48(1):11-19, 2010.

Prieto, L.; Robles, E.; Salazar, L. M. \& Daniel, E. Burnout en médicos de atención primaria de la provincia de Cáceres. Aten. Prim., 29(5):294-302, 2002.
Quiroz, R. \& Saco, S. Factores asociados al Síndrome Burnout en médicos y enfermeras del Hospital Nacional Sur-Este de EsSalud del cusco. SITUA, XXIII:11-22, 1999.

Rajab, E. Effects of stress in dentists students. J. Dent. Res., 69(2):489-499, 2001.

Robles Velásquez, R. \& Cáceres Gutiérrez, L. Síndrome Burnout en cirujanos dentistas de hospitales de Lima Metropolitana. Odontol. Sanmarquina, 13(1):710, 2010.

Sanders, E. Prevention and differences among dental office personal. Gen. Dent., 66(9):1021-30, 2002.

Sánchez-Madrid, M. A.; Delgado-Martínez, A. D. \& Alcalde Pérez, D. Prevalencia del síndrome de burnout en los cirujanos ortopédicos de España. Rev. Ortop. Traumatol, 49(5):364-7, 2005.

Seisdedos, N. MBI. Inventario "burnout" de mas-lach. Síndrome del "quemado" por estrés laboral asistencial. Madrid, TEA Ediciones S.A., 1997.

Simón García, M. J.; Blesa Malpica, A. L.; Bermejo Pablos, C.; Calvo Gutierro, M. A. \& Gómez De Enterría Pérez, C. Estresores laborales y satisfacción en la enfermería de una unidad de críticos. Enferm. Intensiva, 16(1):3-14, 2005.

Varela-Centelles, P. I.; Fontao Valcárcel, L. F.; Martínez González, A. M.; Pita Babío, A. \& Valín Liz, M. C. Professional burnout in dentists and stomatologists of the Galician Health Service. Aten. Primaria, 35(6):301-5, 2005.

Dirección para correspondencia:

Angel Visoso Salgado

Profesor Investigador del Centro de Investigación y

Estudios Avanzados en Odontología

Facultad de Odontología

Universidad Autónoma del Estado de México

MÉXICO

Email: avisosos@uaemex.mx

Recibido : 30-01-2012

Aceptado: 14-05-2012 